\title{
Persistent microbial dysbiosis in preterm premature rupture of membranes from onset until delivery
}

Elizabeth A Baldwin, Marina Walther-Antonio, Allison M MacLean, Daryl M Gohl, Kenneth B Beckman, Jun Chen, Bryan White, Douglas J Creedon, Nicholas Chia

Background. Preterm Premature Rupture of Membranes (PPROM) is a major leading cause of preterm births. While the cause for PPROM remains unidentified, it is anticipated to be due to subclinical infection, since a large proportion of PPROM patients display signs of chorioamnionitis. Since subclinical infections can be facilitated by dysbiosis, our goal was to characterize the vaginal microbiome and amniotic fluid discharge upon PPROM, through latency antibiotic treatment, and until delivery, to detect the presence of pathogens, microbiota alteration, and microbial response to treatment.

Methods. Enrolled subjects (15) underwent routine institutional antenatal care for PPROM, including the administration of latency antibiotics. Serial vaginal swabs were obtained from diagnosis of PPROM through delivery and the sequencing of the V3-V5 region of the $16 \mathrm{~S}$ rRNA gene was performed for all collected samples.

Results. The results show that Lactobacilli species were markedly decreased when compared to vaginal swabs collected from uncomplicated pregnancy subjects with a matched gestational time. Prevotella and Peptoniphilus were the most prevalent taxa in PPROM subjects at presentation. The vaginal microbiome of the PPROM subjects varied substantially intra- and inter-subjects. Several taxa were found to be significantly reduced during and after the antibiotic treatment: Weeksella, Lachnospira, Achromobacter, and Pediococcus. In contrast, Peptostreptococcus and Tissierellaceae ph2 displayed a significant increase after the antibiotic treatment. However, the relative abundance of Lactobacillus, Prevotella, and Peptoniphilus was not substantially impacted during the hospitalization of the PPROM subjects. The deficiency of Lactobacillus, and constancy of known pathogenic species, such as Prevotella and Peptoniphilus during and after antibiotics, highlights the persistent dysbiosis and warrants further investigation into mitigating approaches.

Discussion: PPROM is responsible for one third of all preterm births. It is thought that subclinical infection is a crucial factor in the pathophysiology of PPROM because $25-40 \%$ of patients present signs of chorioamnionitis on amniocentesis. Here we sought to directly assess the bacterial content of the vagina and leaking amniotic fluid of subjects at presentation, throughout treatment and up until delivery, in order to search for common 
pathogens, microbiota changes, and microbial response to latency antibiotic treatment. We have found that the vaginal microbiome of PPROM subjects is highly variable and displays significant changes to treatment. However, the unchanging deficiency of Lactobacillus, and persistence of known pathogenic species, such as Prevotella and Peptoniphilus from presentation, through antibiotic treatment and up until delivery, highlights the persistent dysbiosis and warrants further investigation into mitigating approaches. 
1

2

3

4 Elizabeth Baldwin, $\mathrm{MD}^{1,+}$, Marina R.S. Walther-António, $\mathrm{PhD}^{2,+}$, Allison M. MacLean, BS ${ }^{3}$,

5 Daryl Gohl, $\mathrm{PhD}^{3}$, Kenneth B. Beckman, $\mathrm{PhD}^{3}$, Jun Chen, $\mathrm{PhD}^{4}$, Bryan A. White, $\mathrm{PhD}^{5,6}$,

6 Douglas J. Creedon, $\mathrm{MD}, \mathrm{PhD}^{1, *}$, Nicholas Chia, $\mathrm{PhD}^{2,7, *}$

7

$8{ }^{1}$ Obstetrics and Gynecology, Mayo Clinic, Rochester, MN, USA

$9 \quad{ }^{2}$ Department of Surgical Research, Mayo Clinic, Rochester, MN, USA

$10{ }^{3}$ Genomics Center, University of Minnesota, Minneapolis, MN, USA

$11{ }^{4}$ Biomedical Statistics and Informatics, Mayo Clinic, Rochester, MN, USA

$12{ }^{5}$ Carl R. Woese Institute for Genomic Biology, University of Illinois at Urbana-Champaign,

13 Urbana, IL, USA

\section{Persistent Microbial Dysbiosis in Preterm Premature Rupture of Membranes from}

\section{Onset until Delivery}

${ }^{6}$ Animal Sciences, University of Illinois at Urbana-Champaign, Urbana, IL, USA

${ }^{7}$ Department of Physiology and Biomedical Engineering, Mayo College, Rochester, MN, USA;
+These authors contributed equally:

Baldwin.Elizabeth@,mayo.edu, WaltherAntonio.Marina@mayo.edu

${ }^{*}$ Corresponding authors:

Douglas Creedon ${ }^{1}$

200 First Street SW, Rochester, MN, 55905, USA

Email address: Creedon.Douglas@,mayo.edu 
24 Nicholas Chia $^{2,7}$

25200 First Street SW, Rochester, MN, 55905, USA

26 Email address: Chia.Nicholas@mayo.edu

\begin{abstract}
Background. Preterm Premature Rupture of Membranes (PPROM) is a major leading cause of preterm births. While the cause for PPROM remains unidentified, it is anticipated to be due to subclinical infection, since a large proportion of PPROM patients display signs of chorioamnionitis. Since subclinical infections can be facilitated by dysbiosis, our goal was to characterize the vaginal microbiome and amniotic fluid discharge upon PPROM, through latency antibiotic treatment, and until delivery, to detect the presence of pathogens, microbiota alteration, and microbial response to treatment.

Methods. Enrolled subjects (15) underwent routine institutional antenatal care for PPROM, including the administration of latency antibiotics. Serial vaginal swabs were obtained from diagnosis of PPROM through delivery and the sequencing of the V3-V5 region of the 16S rRNA gene was performed for all collected samples.
\end{abstract}

Results. The results show that Lactobacilli species were markedly decreased when compared to vaginal swabs collected from uncomplicated pregnancy subjects with a matched gestational time. Prevotella and Peptoniphilus were the most prevalent taxa in PPROM subjects at presentation. The vaginal microbiome of the PPROM subjects varied substantially intra- and inter-subjects. Several taxa were found to be significantly reduced during and after the antibiotic treatment: Weeksella, Lachnospira, Achromobacter, and Pediococcus. In contrast, Peptostreptococcus and Tissierellaceae ph2 displayed a significant increase after the antibiotic treatment. However, the 
47 relative abundance of Lactobacillus, Prevotella, and Peptoniphilus was not substantially

48 impacted during the hospitalization of the PPROM subjects. The deficiency of Lactobacillus, and

49

50

51

52

53

54

constancy of known pathogenic species, such as Prevotella and Peptoniphilus during and after antibiotics, highlights the persistent dysbiosis and warrants further investigation into mitigating approaches.

Discussion. PPROM is responsible for one third of all preterm births. It is thought that subclinical infection is a crucial factor in the pathophysiology of PPROM because $25-40 \%$ of patients present signs of chorioamnionitis on amniocentesis. Here we sought to directly assess the bacterial content of the vagina and leaking amniotic fluid of subjects at presentation, throughout treatment and up until delivery, in order to search for common pathogens, microbiota changes, and microbial response to latency antibiotic treatment. We have found that the vaginal microbiome of PPROM subjects is highly variable and displays significant changes to treatment. However, the unchanging deficiency of Lactobacillus, and persistence of known pathogenic species, such as Prevotella and Peptoniphilus from presentation, through antibiotic treatment and up until delivery, highlights the persistent dysbiosis and warrants further investigation into mitigating approaches.

Preterm birth is the single leading cause of neonatal morbidity and mortality in the developed world, and despite efforts to identify causes, preventative strategies and treatment options, the incidence of preterm birth continues to rise in the United States(Goldenberg et al. 2008). Preterm premature rupture of membranes (PPROM) directly causes one third of all preterm births (Mercer et al. 2000). Subclinical infection likely plays a key role in the 
70 pathophysiology of PPROM and subsequent onset of preterm labor, as evidenced by the fact that

$7125-40 \%$ of patients with PPROM present signs of chorioamnionitis on amniocentesis (Simhan \&

72 Canavan 2005). Furthermore, specific microbes associated with bacterial vaginosis (BV) such as

73 Gardnerella vaginalis and Mycoplasma hominis have been linked to pregnancy complications,

74

including PPROM, preterm birth (Leitich et al. 2003); (McDonald et al. 1991); (Romero et al. 2002); (Hay et al. 1994), and intra-amniotic infections (Mendz et al. 2013). Attempts to prevent preterm birth by using antibiotics prior to the onset of labor have not been very efficacious (Oliver \& Lamont 2013); (Hauth et al. 1995); (Brocklehurst et al. 2013). This suggests that our current knowledge of the vaginal microbes associated with adverse pregnancy outcomes, mainly based on clinical and traditional culture techniques remains incomplete (White et al. 2011).

Recently, the advent of next generation sequencing techniques such as 16S rRNA gene hypervariable tag sequencing has allowed a more complete characterization of the vaginal microbial ecology also known as the microbiome. Studies using 16S rRNA gene technology have demonstrated a broad spectrum of microbial organisms not previously identified in the vagina using traditional culture techniques (Fredricks et al. 2005); (Zhou et al. 2004); (Ravel et al. 2010). In studies of healthy non-pregnant women, the vaginal microbiome is characterized by five community profiles or subtypes most primarily dominated by a mixture of Lactobacillus species (Ravel et al. 2010). During normal pregnancy, this dominance broadens and the microbial diversity undergoes a marked decrease with convergence toward a single subtype (Aagaard et al. 2012); (Romero et al. 2014b); (Walther-António et al. 2014). Although, the vaginal microbiome changes in relative abundance of taxa experienced by women that delivered at term has not been shown to be significantly different from women that delivered preterm (Romero et al. 2014a), higher absolute abundances of particular microorganisms such as 
93

94

95

96

97

98

99

100

101

102

103

104

105

106

107

108

109

110

111

112

\section{Ethics Statement}

114 Subjects were consented under IRB \#12-001675, which was reviewed and approved by the Mayo

115 Clinic Institutional Review Board. All subjects provided written consent. 


\section{Subjects Enrollment}

118 Here we report the results from 15 subjects enrolled upon admission for PPROM to the Mayo

119 Clinic Hospital - Rochester. Inclusion criteria consisted of the following: age >18 years; no

120 known pregnancy complications prior to admission; ability to provide written informed consent;

121 willingness to participate in mandatory translational research component of the study; weight

122 greater than 50 kilograms; and confirmed preterm rupture of membranes based on clinical

123 criteria. Exclusion criteria consisted of the following: known immunodeficiency; chronic active

124 viral infection (including HIV, HTLV and hepatitis); known autoimmune disease; solid organ or

125 transplant recipient; and multiple gestations. Upon enrollment, participations were requested to

126 fill out a questionnaire detailing sexual and reproductive health history and hygiene practices.

127 Selected questionnaire data is available in Supplemental Table 1. Metadata from the

128 questionnaires was stored using REDCap (Harris et al. 2009). Relevant medical information

129 related to the time of labor and delivery is also included in Supplemental Table 1. All subjects

130 were managed according to the standard institutional protocol for PPROM. This included

131 hospitalization, administration of a course of antenatal steroids if $<34$ weeks gestation on

132 admission and a standardized course of latency antibiotics (12 subjects received

133 ampicillin/amoxicillin and azithromycin; two subjects received clindamycin and azithromycin

134 due to penicillin allergy; and one subject received penicillin alone due to presentation in active

135 labor). Delivery was undertaken for spontaneous preterm labor, nonreassuring maternal or fetal

136 status, clinical concern for infection, or when a gestational age of 34 weeks was reached.

\section{Sample Collection}


139 Dacron swabs were collected during sterile speculum exam performed by an obstetrician from

140 the posterior vaginal fornix and placed in a Nucleic Acid Transport collection tube. After

141 collection the samples were stored at $-80^{\circ} \mathrm{C}$ until processing. Vaginal samples were collected at

142 the time of admission, every three days for the first two weeks of admission and weekly

143 thereafter until delivery. An additional vaginal sample was collected at the time of delivery and

144 a swab from the fetal surface of the placental membranes after delivery for 6 of the 15 subjects.

145

\section{Sample Processing}

147 Samples were thawed and centrifuged for 10 minutes at $10000 \mathrm{~g}$ to collect the bacterial cells, and the supernatant was discarded. Genomic DNA extraction was performed by using the MoBio Ultraclean Soil Kit (MoBio Laboratories, Inc., Carlsbad, CA); with the MP FastPrep (MP Biomedicals, Solon, $\mathrm{OH}$ ) for 40 seconds at $6.0 \mathrm{~m} / \mathrm{s}$. Incubation period was done for a minimum of 30 minutes. After extraction the DNA content was measured using High Sensitivity Qubit (Life Technologies Corporation, Carlsbad, CA) with the results ranging from below detection to $23 \mathrm{ng} / \mathrm{ul}$ of DNA. The V3-V5 region of the 16S rRNA was then amplified using a two-step polymerase chain reaction (PCR) protocol. The primary amplification was done using the following recipe: $3 \mu 1$ template DNA, $0.5 \mu 1$ nuclease-free water, $1.2 \mu 1$ 5x KAPA HiFi buffer (Kapa Biosystems, Woburn, MA), $0.18 \mu 110$ mM dNTPs (Kapa Biosystems, Woburn, MA), 0.3 $\mu 1$ DMSO (Fisher Scientific, Waltham, MA), $0.12 \mu 1$ ROX (25 $\mu \mathrm{M})$ (Life Technologies, Carlsbad, CA), $0.003 \mu 1$ 2000x SYBR Green, $0.12 \mu 1$ KAPA HiFi Polymerase (Kapa Biosystems, Woburn, MA), $0.3 \mu 1$ forward primer $(10 \mu \mathrm{M}), 0.3 \mu 1$ reverse primer $(\mu 1)$. The following cycling conditions were used: $95^{\circ} \mathrm{C}$ for 5 minutes, followed by 25 cycles of $98^{\circ} \mathrm{C}$ for 16120 seconds, $55^{\circ} \mathrm{C}$ for 15 seconds, $72^{\circ} \mathrm{C}$ for 1 minute. The primers for the primary amplification 
162 contained both $16 \mathrm{~S}$-specific primers (357F and 926R), as well as adapter tails for adding indices

163 in a secondary amplification. The primer sequences for the primary amplification were as

164 follows (16S-specific sequences in bold):

165 V3F_Nextera:

166 TCGTCGGCAGCGTCAGATGTGTATAAGAGACAGCCTACGGGAGGCAGCAG

167 V5R_Nextera:

168 GTCTCGTGGGCTCGGAGATGTGTATAAGAGACAGCCGTCAATTCMTTTRAGT

169 Next, these amplicons were diluted 1:100 in sterile, nuclease-free water, and a second PCR

170 reaction was set up to add the Illumina flow cell adapters and indices. The secondary

171 amplification was done using the following recipe: $5 \mu 1$ template DNA, $1 \mu 1$ nuclease-free water,

$1722 \mu 15 x$ KAPA HiFi buffer (Kapa Biosystems, Woburn, MA), $0.3 \mu 110$ mM dNTPs (Kapa

173 Biosystems, Woburn, MA), $0.5 \mu$ 1 DMSO (Fisher Scientific, Waltham, MA), $0.2 \mu 1$ KAPA HiFi

174 Polymerase (Kapa Biosystems, Woburn, MA), $0.5 \mu$ l forward primer $(10 \mu \mathrm{M}), 0.5 \mu 1$ reverse

175 primer $(\mu \mathrm{l})$. The following cycling conditions were used: $95^{\circ} \mathrm{C}$ for 5 minutes, followed by 10

176 cycles of $98^{\circ} \mathrm{C}$ for 20 seconds, $55^{\circ} \mathrm{C}$ for 15 seconds, $72^{\circ} \mathrm{C}$ for 1 minute, followed by a final

177 extension at $72^{\circ} \mathrm{C}$ for 10 minutes. The indexing primers are as follows (X marks the positions of

178 the 8 bp indices):

179 Forward indexing primer:

AATGATACGGCGACCACCGAGATCTACACXXXXXXXXTCGTCGGCAGCGTC

181 Reverse indexing primer:

CAAGCAGAAGACGGCATACGAGATXXXXXXXXGTCTCGTGGGCTCGG

183 The products of the amplification were quantified using a PicoGreen dsDNA assay (Life

184 Technologies, Carlsbad, CA), and the samples were normalized, pooled, and approximately $1 \mu \mathrm{g}$ 
185 of material was concentrated to $10 \mu \mathrm{l}$ using 1.8x AMPureXP beads (Beckman Coulter, Brea,

186 CA). The pooled sample was then size selected at $723 \mathrm{bp}+/-20 \%$ on a Caliper XT DNA 750

187 chip (Caliper Life Science, Hopkinton, MA). The size-selected material was cleaned up using

188 AMPureXP beads, and eluted in $20 \mu \mathrm{l}$ of EB buffer (10 mM Tris- $\mathrm{HCl}, \mathrm{pH} 8.5)$. The final pooled

189 sample was quantified using the PicoGreen dsDNA assay, and analyzed using an Agilent

190 Bioanalyzer High Sensitivity Chip (Agilent Technologies, Santa Clara, CA). Finally, the sample

191 pool was diluted to $2 \mathrm{nM}$ based on the PicoGreen measurements, and $10 \mu 1$ of the $2 \mathrm{nM}$ pool was

192 denatured with $10 \mu \mathrm{l}$ of $0.2 \mathrm{~N} \mathrm{NaOH}$, diluted to $8 \mathrm{pM}$ in Illumina's HT1 buffer, spiked with $20 \%$

193 phiX, heat denatured at $96^{\circ} \mathrm{C}$ for 2 minutes, and sequenced using a MiSeq 600 cycle v3 kit

194 (Illumina, San Diego, CA).

195

196

\section{Processing of Controls}

197 Control samples consisted of five "empty collection” replicates. In brief, five swabs were

198

199

200

201

202

203

204

205

206

207

immersed in the collection buffer and DNA was extracted, amplified, and sequenced using the same procedures and reagents used for the PPROM samples. In addition, three other controls were performed: 1) one extraction with only the collection buffer was performed (no swab), 2) a PCR negative control was sequenced, 3) a positive control of a pure isolate of Campylobacter jejuni. There was no detectable amplification in the negative controls by qPCR. The positive control (Campylobacter jejuni) was carried forward to sequencing as well to guarantee that the negative controls were sequenced at comparable depth. The eight control samples were spiked into a MiSeq run at a comparable per-sample concentration to that of the PPROM sample MiSeq run. As expected, the extraction and water negative controls yielded much lower numbers of reads (between $0.0084 \%$ and $0.0406 \%$ of the total reads in the run). 
209 Sequencing Processing and Outcome

210 To remove the 5'-Adapter-primer sequence on forward reads which has a total length of $58 \mathrm{bps}$,

211 for each forward read, we took $60 \mathrm{bps}$ from the 5' end and BLAST against the 5'-Adapter-primer

212 sequence allowing one base mismatch, and the matched region of the reads were removed if

213 present at the beginning of the sequence read. To remove the 5'-Adapter-barcode-primer

214 sequence on reverse reads which has a total length of $65 \mathrm{bps}$, we first generated a group of

215 sequences, through a combination of different barcodes and wobbly bases. There are a total of 73

216 samples taken from 15 subjects, each sample with a unique barcode. And there are two wobbly

217 bases $\mathrm{M}$ and $\mathrm{G}$ in the sequence, each of which denotes an alternative base of $\mathrm{A}$ or $\mathrm{C}$ and $\mathrm{A}$ or $\mathrm{G}$

218 respectively. Thus the total number of sequence combinations is $77 \times 2 \times 2=292$. We created a

219 BLAST database from the 292 sequences. Then, for each reverse read, we took $67 \mathrm{bps}$ from the

220 5' end and BLAST against the created database allowing one base mismatch except for the

221 barcode region. The matched region of each read was removed if present at the beginning of the

222 sequence read and the samples were de-multiplexed by the matched barcode on each read. Due

223 to the low number of R2 samples passing quality control (at least $187 \mathrm{bp}$ per sequence read and a

224 minimum of 2,000 sequence reads per sample), only R1 reads were used for analysis. A total of

$2255,575,178 \mathrm{R} 1$ sequence reads $(5,527$ to 174,479 sequence reads per sample) passed quality

226 control.

227

228

Sequence Analysis 
229 Sequence reads were aligned with our own custom multiple alignment tool known as the Illinois-

230 Mayo Taxon Operations for RNA Dataset Organization (IM-TORNADO) that merges paired end

231 reads into a single multiple alignment and obtains taxa calls (Sipos et al. 2010). Operational

232 taxonomic units were clustered using UPARSE (Edgar 2013). Further processing for

233 visualization and statistical analysis was performed using QIIME (Caporaso et al. 2010). To

234 identify differential abundant bacteria genera, we fit a linear regression model to the square-root

235 transformed genus proportion data. We used the t-statistic as the test statistic. To address the

236 non-normality of the outcome variable as well as within-subject correlation, we used permutation

237 to assess the statistical significance. Permutation was performed 1,000 times and was

238 constrained within the subject to retain the original correlation structure. We also fit a

239 generalized mixed effects model to the genus presence/absence data using PQL method,

240 assuming a random intercept for each subject. Statistical significance was assessed based on

241 Wald test. False discovery control (B-H procedure) was used for correcting multiple testing.

242 Sequences are publicly available at SRA, study accession: SRP061714. The taxonomic

243 assignments are provided in Supplemental Table 2.

\section{Results}

Our cohort consisted of 15 subjects (Table 1; age range: 19-37 years old; Mean \pm

247 Standard Deviation: $29 \pm 4$ years old). The majority were Caucasian (14), and one was East

248 Asian. Within the cohort, there were no complications specific to pregnancy prior to or

249 subsequent to diagnosis of PPROM including no diagnoses of gestational diabetes or

250 hypertensive disorders of pregnancy. The cohort included 10 parous and 5 nulliparous women, 251 with four subjects having a history of preterm delivery with a previous pregnancy. From these 15 
252 subjects, a total of 61 vaginal samples and 6 placental samples were collected (Fig 1; selected 253 metadata associated with each subject can be found in Table S1).

254 PPROM Before Antibiotic Treatment. In order to assess the näive maternal microbiome at time 255 of presentation with PPROM, we examined the admission samples from nine subjects that were 256 obtained before the administration of antibiotics and compared them with those of a companion 257 study, where 12 women experiencing an uncomplicated pregnancy were sampled longitudinally 258 (Walther-António et al. 2014). Using gestational age-matched samples from the companion study we compared the vaginal microbiome taxonomic assignments to the PPROM subjects. The microbiome in the PPROM subjects exhibited a diversity of taxa that was in stark contrast to the normal pregnancy subjects (Fig 2), and was marked by an underrepresentation of lactobacilli (Fig 3). Overall, the most common pathogens detected in the PPROM subjects before the administration of antibiotics were Prevotella and Peptoniphilus (Fig 4).

PPROM During and After Antibiotic Treatment. The administration of antibiotics did not significantly impact the relative abundance of Lactobacillus, Prevotella, or Peptoniphilus during or after the antibiotic treatment (Table 2 and Table 3). However, during the administration of the treatment, Weeksella, Lachnospira, Achromonacter and Pediococcus showed a significant decrease, while Peptostreptococcus and Tissierellaceae ph2 showed a significant increase (Table 2 shows presence/absence significance most powerful for low abundance taxa, Table 3 shows proportion significance more powerful for high abundance taxa). We also compared the BrayCurtis (BC) and UniFrac distances (with similar results) to address relevant questions. One of them was whether there was a statistical difference in the vaginal microbiome of subjects that underwent a cesarian-section (C-section) when compared to subjects that had a normal

274 spontaneous vaginal delivery (NSVD). We have 5 subjects that underwent a C-section and 10 
275 with a NSVD. PERMANOVA based on BC distance produced a p-value of 0.23 . Therefore, we 276 do not have enough supporting evidence that the delivery mode affects the microbiome. We

277 further enquired whether parity was a determining factor for the vaginal microbiome. There are 27810 parous subjects (parity $>0$ ) and 5 nulliparous subjects (parity=0). PERMANOVA based on 279 BC distance produced a p-value of 0.20. Therefore, we do not have enough supporting evidence 280 that parity influences the vaginal microbiome.

281 Placental Microbiome. To assess the placental microbiome, we compared the six placental 282 samples with the matched maternal vaginal samples obtained at the time of delivery. The 283 placental microbiome was statistically distinct from the vaginal microbiome (Bray Curtis 284 distance p-value 0.08 - PERMANOVA, within subject 1,000 permutations). However, given the 285 limited amount of samples, there was insufficient statistical power to identify differential taxa.

286 Placental samples showed high individual variability and weak correlation with the maternal 287 vaginal microbiome (Fig 5). The most prevalent taxa recovered were Alicyclobacillus and 288 Corynebacterium. In order to determine if the detected placental microbiome could be the result 289 of contamination from the vaginal microbiome we performed a permutation test and compared 290 the BC and Unifrac distances. There are six subjects that have matched placental and vaginal 291 samples. Two subjects underwent a C-section and four subjects had a NVSD. We do not see that 292 the placental swabs from the vaginal deliveries are significantly closer to the vaginal swabs than 293 the placental swabs from $\mathrm{C}$-sections $(\mathrm{p}=0.53)$. However, one placental swab from a NVSD 294 subject (PPROM_7) has potential contamination indicated by a very small distance $(0.48)$ when 295 compared to the range among the remaining NSVD distances $(0.97-0.99)$ and C-sections $(0.88$ 296 0.91). We also looked into whether the delivery vaginal microbiome was the most similar to the 297 placental swab or if the placental swab was rather closer to a previous vaginal swab from the 
298 same subject. There are four subjects with multiple vaginal swabs and a placental swab. By

299 comparing the distance from the placental swab to the delivery swab, to the distance from the

300 placental swab to other vaginal swabs within the same subject, we see a trend that the

301 placental swab is more similar to the last delivery sample $(\mathrm{p}=0.078$, permutation test, shuffling

302 the vaginal swabs within the same subject).

303 Controls. The sequencing of our controls - Negative PCR control, five quality control (QC)

304 replicates (empty swab collections) and the DNA extraction and amplification of the TE buffer

305 used for the sample collection - showed very little overlap with the major taxa seen in the study

306 samples (Supplemental Table 3).

307

308

\section{Discussion}

309

310

Principal findings of the study. The analysis of the vaginal microbiome of 15 PPROM subjects

311 from presentation, throughout their hospital stay and treatment, and up until delivery, shows that

312 the microbiome is dysbiotic and highly variable between subjects since presentation. The

313 antibiotic treatment administered for the condition did not eliminate the presence of pathogenic

314 species such as Prevotella and Peptoniphilus, which remained until the time of delivery, as did

315 the deficiency in lactobacilli species.

316 Our results also show that the vaginal microbiota of pregnant subjects with PPROM is

317 distinct from that of subjects undergoing an uncomplicated pregnancy. The findings indicate that

318 the vaginal microbiome after PPROM is more diverse and dynamic than previously observed

319 during normal pregnancies (Walther-António et al. 2014). Of specific interest is the difference in

320 lactobacilli abundance in PPROM. Lactobacilli acidify their environment and limit diversity 
321 through a process of niche expansion - allowing for the dominance of lactobacilli in most

322 vaginal microbiota observed to date. The loss of lactobacilli, along with many other factors, may

323 place a key role in the destabilization of the PPROM-associated vaginal microbiome. This

324 depletion may be due to the displacement either by the constant leakage of alkaline amniotic

325 fluid or may precede the PPROM event. It is also worth emphasizing that although the swabs

326 were physically placed in the posterior fornix for the sampling, this was likely to contain varying

327 amounts of amniotic fluid in PPROM subjects. Once there is a rupture of the membranes,

328 amniotic fluid continues to leak from the cervix and when a patient is in the supine position the

329 posterior fornix is where that fluid will collect. The patients remain hospitalized until delivery,

330 and the samples were obtained in the patient's hospital bed. So unless the patient had been

331 ambulating just prior to sampling, there will be some amniotic fluid pooled in the posterior

332 fornix. Once lactobacilli are displaced, opportunistic bacteria may occupy the available niche

333 leading to an unbalanced microbial ecology (dysbiosis) with potentially negative consequences

334 for health. The transient nature of the PPROM microbiome is consistent with the idea of an

335 ecology that is out of equilibrium. Prevotella and Peptoniphilus emerge as taxa of particular

336 interest in PPROM given their prevalence at presentation and persistence throughout treatment,

337 evidence of prominent role in persistent bacterial vaginosis and preterm labor (Marrazzo et al.

338 2008); (Smayevsky et al. 2001); (Wang et al. 2013); (Mikamo et al. 1999) and display of broad-

339 spectrum antibiotic resistance (Sherrard et al. 2013); (Tanaka et al. 2006). The highly variable

340 placental microbiome recovered and the weak correlation with the maternal microbiome raises

341 the possibility that the uterine microbiome may be an independent driver of the placental

342 microenvironment. The formation of the cervical mucus plug as early as seven weeks of

343 gestation provides a mechanical and chemical barrier between the vaginal and uterine 
344 environments (Hein et al. 2002). The influence of the vaginal microbiome in the uterine

345 microbial niche is therefore anticipated to be reduced for as long as the mucus plug is intact. The

346 fact that in this study Prevotella was a prominent pathogen found in the vaginal fluid discharge

347 and placental membranes indicates a very likely role in the etiology of PPROM. The known

348 association of Prevotella with bacterial vaginosis (Hillier et al. 1993) and preterm labor (Holst et

349 al. 1994) strengthen this possibility. It is however, important to note that preterm labor and

350 PPROM are distinct conditions. While PPROM often leads to preterm labor, preterm labor can

351 be caused by a multitude of conditions unrelated to PPROM. It is therefore not surprising that

352 preterm labor studies (Romero et al. 2014a); (Mendz et al. 2013) may or may not align with our

353 findings.

354 Our study is limited by the small number of patients. Another limitation is that the

355 vaginal swabs collected from PPROM subjects likely contained both amniotic fluid and vaginal

356 fluid. We are unable to determine the proportion of amniotic fluid in each sample, which is

357 expected to vary with each collection. By contrast, the vaginal swabs collected from

358 uncomplicated pregnancy subjects are not anticipated to have had a significant presence of

359 amniotic fluid.

360 Despite the low amount of microbial DNA present in the samples, we were able to rule

361 out that the relevant taxa in this study could be the result of contamination. As shown in Table 3

362 we were able to find several well-known contaminants (Salter et al. 2014), but not the taxa

363 relevant to our study with one exception, Corynebacterium. This taxa was predominant in the

364 placenta of our PPROM subjects, which supports previous findings in the placenta of preterm

365 deliveries (Oh et al. 2010). It is possible that its presence may be overemphasized in this study 
366 due to its detection in the collection buffer, but it is undoubtedly present in the study samples as

367 well.

368

369

370

371

372 variability. An interesting area of future research would be to assess the changes in the

373 microbiome noted in our study as a potential causative factor predating diagnosis of PPROM by

374

longitudinal assessment of the vaginal microbiome in pregnant women at high risk for preterm

375

376

377 premature rupture of membranes.

\section{Conclusions}

The deficiency in lactobacilli species and persistence of known pathogenic species at admission, and during and after antibiotics, highlights a marked dysbiosis with high individual

6

(

7

\section{Acknowledgments}

The authors wish to acknowledge clinical, diagnostic and clerical staff at Mayo Clinic.

\footnotetext{
Aagaard K, Riehle K, Ma J, Segata N, Mistretta T-A, Coarfa C, Raza S, Rosenbaum S, Van den Veyver I, and Milosavljevic A. 2012. A metagenomic approach to characterization of the vaginal microbiome signature in pregnancy. PLoS One 7:e36466.

Brocklehurst P, Gordon A, Heatley E, and Milan SJ. 2013. Antibiotics for treating bacterial vaginosis in pregnancy. The Cochrane Library.

Caporaso JG, Kuczynski J, Stombaugh J, Bittinger K, Bushman FD, Costello EK, Fierer N, Pena AG, Goodrich JK, and Gordon JI. 2010. QIIME allows analysis of high-throughput community sequencing data. Nature methods 7:335-336.

Edgar RC. 2013. UPARSE: highly accurate OTU sequences from microbial amplicon reads. Nature methods 10:996-998.

Epstein FH, Parry S, and Strauss JF. 1998. Premature rupture of the fetal membranes. New England Journal of Medicine 338:663-670.

Flynn CA, Helwig AL, and Meurer LN. 1997. Bacterial vaginosis in pregnancy and the risk of prematurity: a meta-anaysis. Medical College of Wisconsin.

Fredricks DN, Fiedler TL, and Marrazzo JM. 2005. Molecular identification of bacteria associated with bacterial vaginosis. New England Journal of Medicine 353:1899-1911.
} 
396

397

398

399

400

401

402

403

404

405

406

407

408

409

410

411

412

413

414

415

416

417

418

419

420

421

422

423

424

425

426

427

428

429

430

431

432

433

434

435

436

437

438

439

440

441

Goldenberg RL, Culhane JF, Iams JD, and Romero R. 2008. Epidemiology and causes of preterm birth. The Lancet 371:75-84.

Harris PA, Taylor R, Thielke R, Payne J, Gonzalez N, and Conde JG. 2009. Research electronic data capture (REDCap) - a metadata-driven methodology and workflow process for providing translational research informatics support. Journal of biomedical informatics 42:377-381.

Hauth JC, Goldenberg RL, Andrews WW, DuBard MB, and Copper RL. 1995. Reduced incidence of preterm delivery with metronidazole and erythromycin in women with bacterial vaginosis. New England Journal of Medicine 333:1732-1736.

Hauth JC, MacPherson C, Carey JC, Klebanoff MA, Hillier SL, Ernest J, Leveno KJ, Wapner R, Varner M, and Trout W. 2003. Early pregnancy threshold vaginal $\mathrm{pH}$ and Gram stain scores predictive of subsequent preterm birth in asymptomatic women. American journal of obstetrics and gynecology 188:831-835.

Hay PE, Lamont RF, Taylor-Robinson D, Morgan DJ, Ison C, and Pearson J. 1994. Abnormal bacterial colonisation of the genital tract and subsequent preterm delivery and late miscarriage. Bmj 308:295-298.

Hein M, Valore EV, Helmig RB, Uldbjerg N, and Ganz T. 2002. Antimicrobial factors in the cervical mucus plug. American journal of obstetrics and gynecology 187:137-144.

Hillier SL, Krohn MA, Rabe LK, Klebanoff SJ, and Eschenbach DA. 1993. The normal vaginal flora, H2O2-producing lactobacilli, and bacterial vaginosis in pregnant women. Clinical Infectious Diseases 16:S273-S281.

Holst E, Goffeng AR, and Andersch B. 1994. Bacterial vaginosis and vaginal microorganisms in idiopathic premature labor and association with pregnancy outcome. Journal of clinical microbiology 32:176-186.

Hutzal CE, Boyle EM, Kenyon SL, Nash JV, Winsor S, Taylor DJ, and Kirpalani H. 2008. Use of antibiotics for the treatment of preterm parturition and prevention of neonatal morbidity: a metaanalysis. American journal of obstetrics and gynecology 199:620. e621620. e628.

Kenyon S, Boulvain M, and Neilson JP. 2013. Antibiotics for preterm rupture of membranes. The Cochrane Library.

Leitich H, Bodner-Adler B, Brunbauer M, Kaider A, Egarter C, and Husslein P. 2003. Bacterial vaginosis as a risk factor for preterm delivery: a meta-analysis. American journal of obstetrics and gynecology 189:139-147.

Marrazzo JM, Thomas KK, Fiedler TL, Ringwood K, and Fredricks DN. 2008. Relationship of specific vaginal bacteria and bacterial vaginosis treatment failure in women who have sex with women. Annals of internal medicine 149:20-28.

McDonald H, O'LOUGHLIN J, Jolley P, Vigneswaran R, and McDonald P. 1991. Vaginal infection and preterm labour. BJOG: An International Journal of Obstetrics \& Gynaecology 98:427-435.

Mendz GL, Kaakoush NO, and Quinlivan JA. 2013. Bacterial aetiological agents of intraamniotic infections and preterm birth in pregnant women. Frontiers in cellular and infection microbiology 3.

Mercer BM, Goldenberg RL, Meis PJ, Moawad AH, Shellhaas C, Das A, Menard MK, Caritis SN, Thurnau GR, and Dombrowski MP. 2000. The preterm prediction study: prediction of preterm premature rupture of membranes through clinical findings and ancillary testing. American journal of obstetrics and gynecology 183:738-745. 
442

443

444

445

446

447

448

449

450

451

452

453

454

455

456

457

458

459

460

461

462

463

464

465

466

467

468

469

470

471

472

473

474

475

476

477

478

479

480

481

482

483

484

485

486
Mikamo H, Kawazoe K, Sato Y, and Tamaya T. 1999. Elastase activity of anaerobes isolated from amniotic fluid with preterm premature rupture of membranes. American journal of obstetrics and gynecology 180:378-380.

Nelson DB, Hanlon A, Nachamkin I, Haggerty C, Mastrogiannis DS, Liu C, and Fredricks DN. 2014. Early Pregnancy Changes in Bacterial Vaginosis-Associated Bacteria and Preterm Delivery. Paediatric and perinatal epidemiology 28:88-96.

Oakley BB, Fiedler TL, Marrazzo JM, and Fredricks DN. 2008. Diversity of human vaginal bacterial communities and associations with clinically defined bacterial vaginosis. Applied and Environmental Microbiology 74:4898-4909.

Oh KJ, Lee KA, Sohn Y-K, Park C-W, Hong J-S, Romero R, and Yoon BH. 2010. Intraamniotic infection with genital mycoplasmas exhibits a more intense inflammatory response than intraamniotic infection with other microorganisms in patients with preterm premature rupture of membranes. American journal of obstetrics and gynecology 203:211. e211211. e218.

Oliver R, and Lamont R. 2013. Infection and antibiotics in the aetiology, prediction and prevention of preterm birth. Journal of Obstetrics \& Gynaecology 33:768-775.

Ravel J, Gajer P, Abdo Z, Schneider GM, Koenig SS, McCulle SL, Karlebach S, Gorle R, Russell J, and Tacket CO. 2010. Vaginal microbiome of reproductive-age women. Proceedings of the National Academy of Sciences:201002611.

Romero R, Espinoza J, Chaiworapongsa T, and Kalache K. 2002. Infection and prematurity and the role of preventive strategies. Seminars in Neonatology: Elsevier. p 259-274.

Romero R, Hassan SS, Gajer P, Tarca AL, Fadrosh DW, Bieda J, Chaemsaithong P, Miranda J, Chaiworapongsa T, and Ravel J. 2014a. The vaginal microbiota of pregnant women who subsequently have spontaneous preterm labor and delivery and those with a normal delivery at term. Microbiome 2:18.

Romero R, Hassan SS, Gajer P, Tarca AL, Fadrosh DW, Nikita L, Galuppi M, Lamont RF, Chaemsaithong P, and Miranda J. 2014b. The composition and stability of the vaginal microbiota of normal pregnant women is different from that of non-pregnant women. Microbiome 2:4.

Salter SJ, Cox MJ, Turek EM, Calus ST, Cookson WO, Moffatt MF, Turner P, Parkhill J, Loman NJ, and Walker AW. 2014. Reagent and laboratory contamination can critically impact sequence-based microbiome analyses. BMC biology 12:87.

Sherrard LJ, Graham KA, McGrath SJ, McIlreavey L, Hatch J, Muhlebach MS, Wolfgang MC, Gilpin DF, Elborn JS, and Schneiders T. 2013. Antibiotic resistance in Prevotella species isolated from patients with cystic fibrosis. Journal of Antimicrobial Chemotherapy:dkt191.

Simhan HN, and Canavan TP. 2005. Preterm premature rupture of membranes: diagnosis, evaluation and management strategies. BJOG: An International Journal of Obstetrics \& Gynaecology 112:32-37.

Sipos M, Jeraldo P, Chia N, Qu A, Dhillon AS, Konkel ME, Nelson KE, White BA, and Goldenfeld N. 2010. Robust computational analysis of rRNA hypervariable tag datasets. PLoS One 5:e15220.

Smayevsky J, Canigia LF, Lanza A, and Bianchini H. 2001. Vaginal microflora associated with bacterial vaginosis in nonpregnant women: reliability of sialidase detection. Infectious diseases in obstetrics and gynecology 9:17-22. 
487

488

Soraisham AS, Singhal N, McMillan DD, Sauve RS, Lee SK, and Network CN. 2009. A multicenter study on the clinical outcome of chorioamnionitis in preterm infants. American journal of obstetrics and gynecology 200:372. e371-372. e376.

Tanaka K, Mikamo H, Nakao Ki, and Watanabe K. 2006. In vitro antianaerobic activity of DX619, a new des-fluoro (6) quinolone. Antimicrobial agents and chemotherapy 50:39083913.

Walther-António MR, Jeraldo P, Miller MEB, Yeoman CJ, Nelson KE, Wilson BA, White BA, Chia N, and Creedon DJ. 2014. Pregnancy's Stronghold on the Vaginal Microbiome. PLoS One 9:e98514.

Wang X, Buhimschi CS, Temoin S, Bhandari V, Han YW, and Buhimschi IA. 2013. Comparative microbial analysis of paired amniotic fluid and cord blood from pregnancies complicated by preterm birth and early-onset neonatal sepsis. PLoS One 8:e56131.

Wen A, Srinivasan U, Goldberg D, Owen J, Marrs CF, Misra D, Wing DA, Ponnaluri S, MilesJay A, and Bucholz B. 2014. Selected vaginal bacteria and risk of preterm birth: an ecological perspective. Journal of Infectious Diseases 209:1087-1094.

White BA, Creedon DJ, Nelson KE, and Wilson BA. 2011. The vaginal microbiome in health and disease. Trends in Endocrinology \& Metabolism 22:389-393.

Zhou X, Bent SJ, Schneider MG, Davis CC, Islam MR, and Forney LJ. 2004. Characterization of vaginal microbial communities in adult healthy women using cultivation-independent methods. Microbiology 150:2565-2573.

\section{Table Legends}

Table 1. Demographics and clinical data for all subjects enrolled. SD - Standard Deviation; Gravid Status - Number of prior pregnancies; Parity - Number of prior deliveries; NSVD Normal Spontaneous Vaginal Delivery. 
$518{ }^{2}$ False discovery control Benjamini-Hochberg (B-H procedure) was used for correcting multiple testing (qvalue $<0.2$ (false discovery rate) 519 considered significant).

520 Table 3. Significant taxa shifts before, during, and after antibiotic treatment.

\begin{tabular}{|c|c|c|c|c|c|c|c|c|c|c|}
\hline & Before & During & After & $\begin{array}{c}\text { Before } \\
\text { vs } \\
\text { During } \\
\end{array}$ & $\begin{array}{l}\text { During } \\
\text { vs After }\end{array}$ & $\begin{array}{l}\text { Before } \\
\text { vs After }\end{array}$ & $\begin{array}{c}\text { Before } \\
\text { vs } \\
\text { During } \\
\end{array}$ & $\begin{array}{l}\text { During } \\
\text { vs After }\end{array}$ & $\begin{array}{l}\text { Before } \\
\text { vs After }\end{array}$ & \multirow[t]{2}{*}{ Signal } \\
\hline $\begin{array}{l}\text { Taxa (Phylum, Classe, } \\
\text { Order, Family, Genus) }\end{array}$ & Mean & Mean & Mean & $p$-value ${ }^{1}$ & $p$-value ${ }^{1}$ & $p$-value ${ }^{1}$ & qvalue $^{2}$ & qvalue $^{2}$ & qvalue $^{2}$ & \\
\hline $\begin{array}{l}\text { Firmicutes, Bacilli, } \\
\text { Lactobacillales, } \\
\text { Lactobacillaceae, } \\
\text { Pediococcus } \\
\end{array}$ & $1.9 \mathrm{E}-03$ & $4.1 \mathrm{E}-03$ & $1.6 \mathrm{E}-05$ & 0.299 & 0.004 & 0.002 & 0.84 & 0.084 & 0.084 & Decrease \\
\hline $\begin{array}{l}\text { Proteobacteria, } \\
\text { Betaproteobacteria, } \\
\text { Burkholderiales, } \\
\text { Alcaligenaceae, } \\
\text { Achromobacter }\end{array}$ & 4.3E-04 & $1.8 \mathrm{E}-02$ & $3.4 \mathrm{E}-06$ & 0.267 & 0.003 & 0.018 & 0.84 & 0.084 & 0.308 & Decrease \\
\hline $\begin{array}{l}\text { Firmicutes, Clostridia, } \\
\text { Clostridiales, } \\
\text { Peptostreptococcaceae, } \\
\text { Peptostreptococcus }\end{array}$ & $2.1 \mathrm{E}-03$ & 2.7E-04 & $1.9 \mathrm{E}-02$ & 0.247 & 0.001 & 0.001 & 0.84 & 0.042 & 0.084 & Increase \\
\hline $\begin{array}{l}\text { Firmicutes, Clostridia, } \\
\text { Clostridiales, } \\
\text { Tissierellaceae, ph2 }\end{array}$ & $1.5 \mathrm{E}-05$ & $0.0 \mathrm{E}+00$ & $1.3 \mathrm{E}-04$ & 0.69 & 0.001 & 0.019 & 0.92 & 0.042 & 0.308 & Increase \\
\hline $\begin{array}{l}\text { Firmicutes, Clostridia, } \\
\text { Clostridiales, } \\
\text { Tissierellaceae, } \\
\text { Peptoniphilus }\end{array}$ & $2.9 \mathrm{E}-02$ & $1.0 \mathrm{E}-02$ & $4.2 \mathrm{E}-03$ & 0.052 & 0.691 & 0.4 & 0.546 & 0.921 & 0.781 & - \\
\hline $\begin{array}{l}\text { Firmicutes, Bacilli, } \\
\text { Lactobacillales, } \\
\text { Lactobacillaceae, } \\
\text { Lactobacillus }\end{array}$ & $2.1 \mathrm{E}-01$ & $1.5 \mathrm{E}-01$ & $1.4 \mathrm{E}-01$ & 0.021 & 0.853 & 0.618 & 0.546 & 0.940 & 0.881 & - \\
\hline $\begin{array}{l}\text { Bacteroidetes, } \\
\text { Bacteroidia, } \\
\text { Bacteroidales, } \\
\text { Prevotellaceae, } \\
\text { Prevotella } \\
\end{array}$ & $7.3 \mathrm{E}-02$ & $1.3 \mathrm{E}-01$ & $1.6 \mathrm{E}-01$ & 0.457 & 0.782 & 0.661 & 0.868 & 0.940 & 0.881 & - \\
\hline
\end{tabular}

${ }^{*}$ A Linear regression model was fit to the square-root transformed genus proportion data. To address the non-normality of the outcome variable as well as within-subject correlation, permutation (1,000 times) was used to assess the statistical significance. Permutation was constrained within each subject to retain the original correlation structure.

${ }^{1}$ Statistical significance was assessed by permutation

${ }^{2}$ False discovery control Benjamini-Hochberg (B-H procedure) was used for correcting multiple testing (qvalue $<0.2$ (false discovery rate) considered significant).

\section{$530 \quad$ Figure Legends}

531 Figure 1. Samples collected and analyzed in the course of the study. 


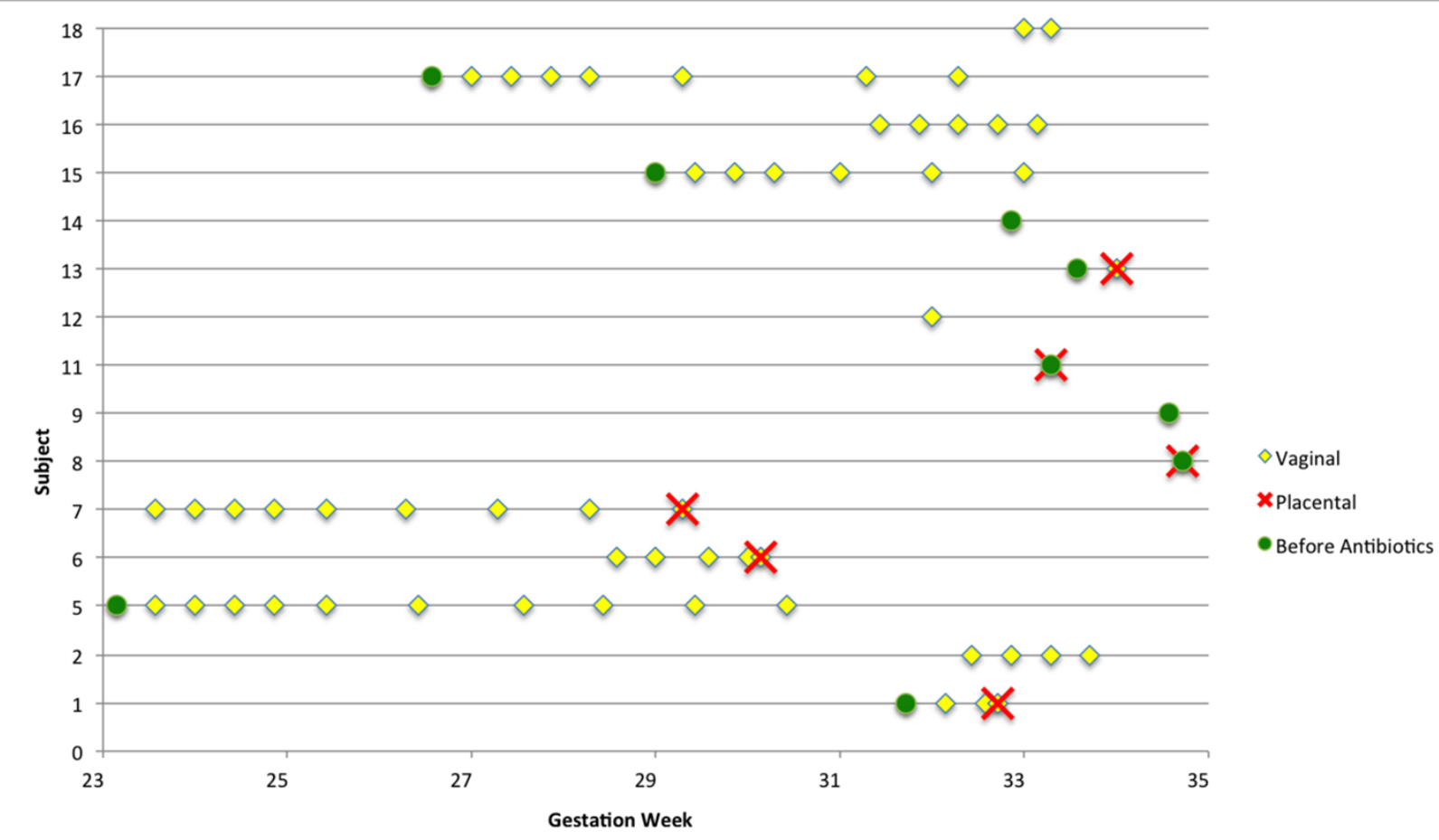

Figure 2. Comparison of relative abundance of taxa at the genus level between vaginal swabs of

534 subjects at the time of presentation at the emergency room with PPROM before the

535 administration of antibiotic treatment ( 9 subjects) and vaginal swabs from subjects that

536 underwent an uncomplicated pregnancy at approximately 29 weeks of gestation (12 subjects).

537 The lack of lactobacilli dominance in PPROM subjects is apparent, as is the inter-individual

538 variation. Only taxa at $>1 \%$ relative abundance in the PPROM subjects are shown for graphical

539 clarity. 


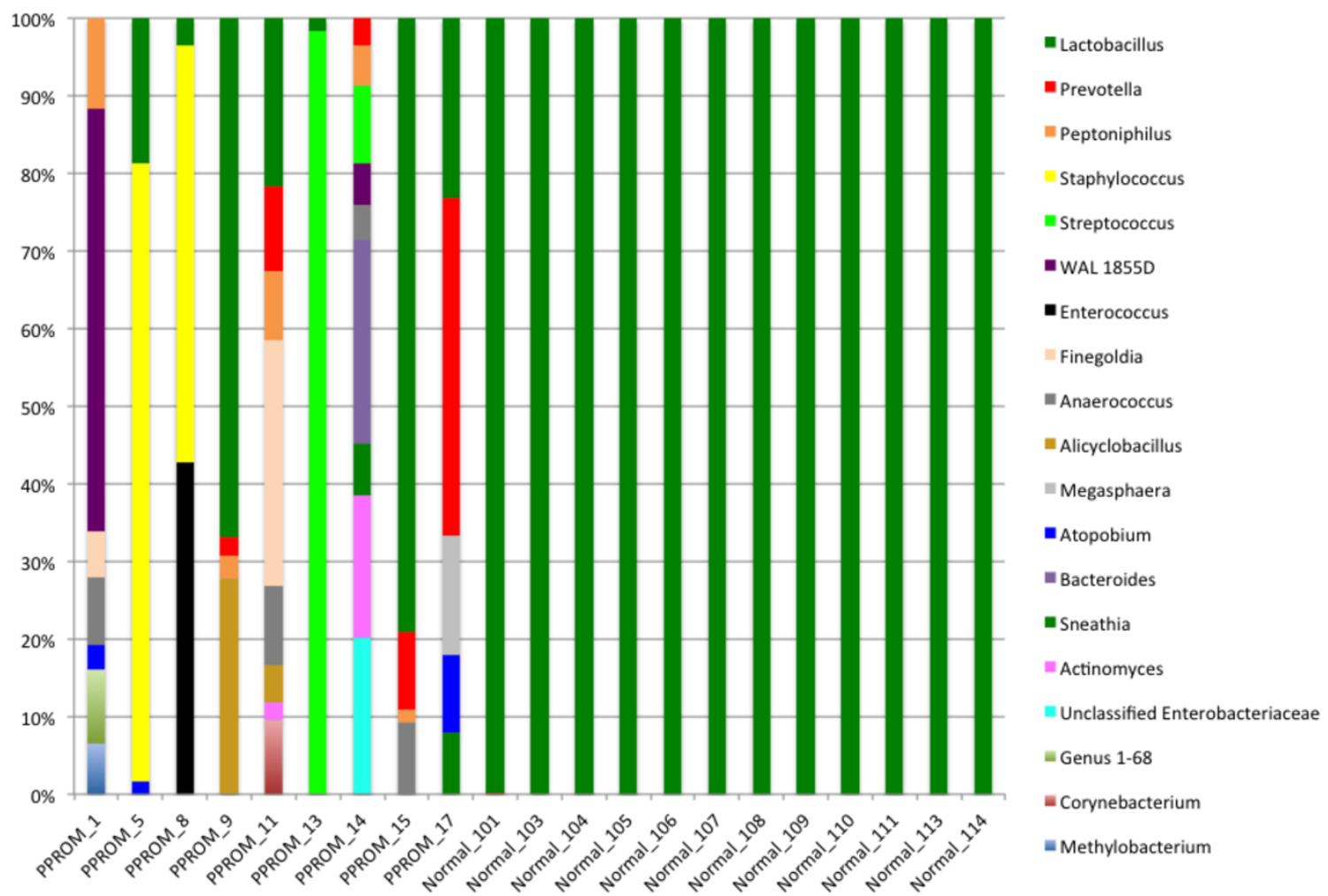

540

Figure 3. Boxplot representing the Lactobacillus frequency in the vaginal swabs of 9 PPROM

subjects at presentation before the administration of antibiotic treatment. Normal is represented by the vaginal swabs of 12 subjects with uncomplicated pregnancies at approximately 29 weeks of gestation. Statistical significance found through linear regression with permutation. 


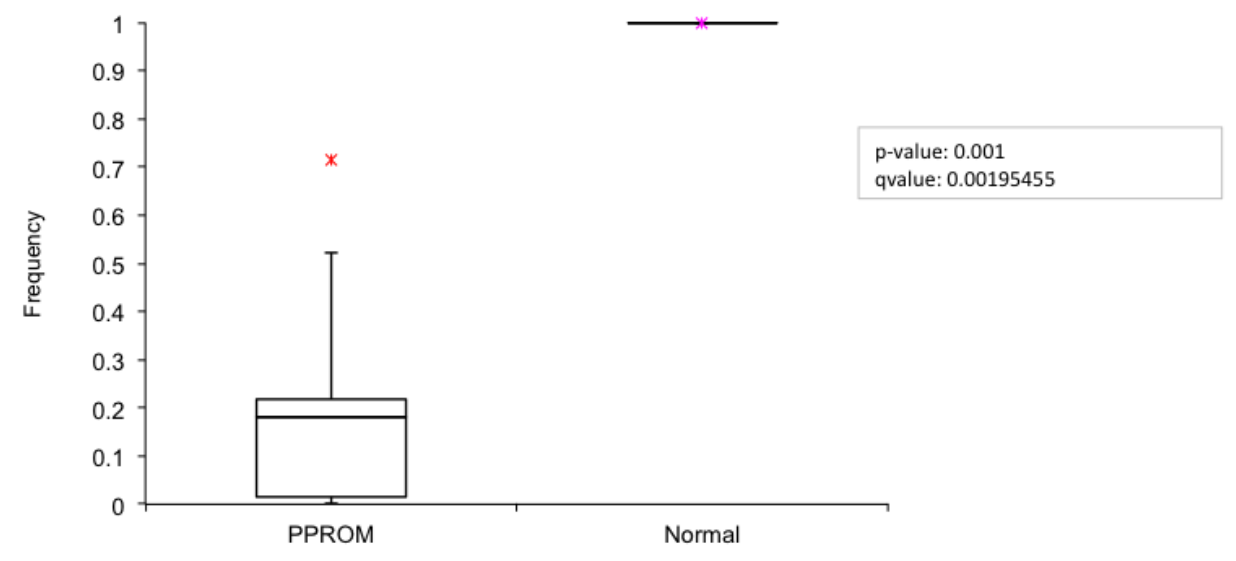

546 Figure 4. Summary (Median $>1 \%$ relative abundance) of taxa at the genus level from all

547 PPROM subjects at presentation before the administration of antibiotic treatment (9 subjects). 


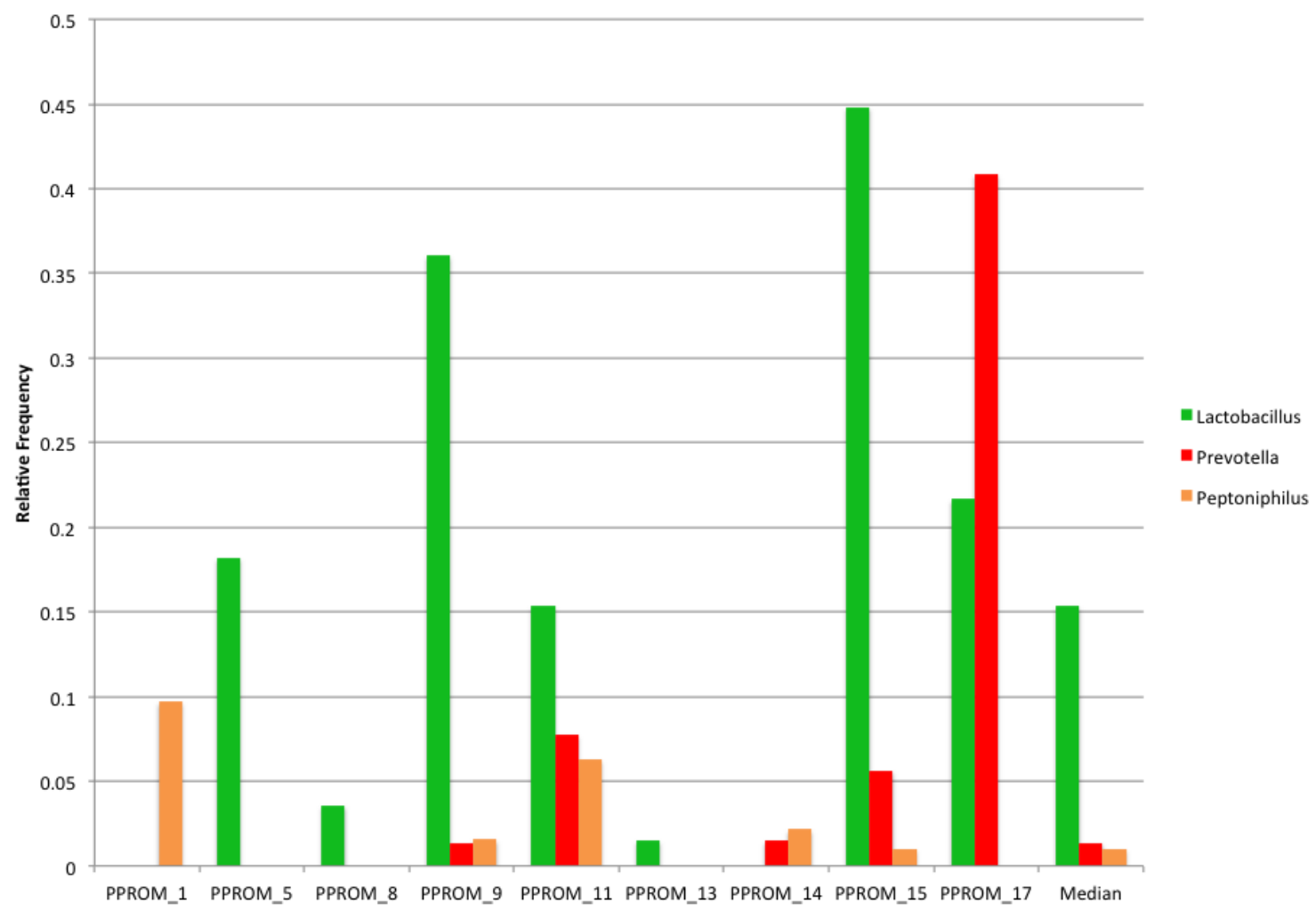

549

550

551

552

Figure 5. Placental and maternal microbiome taxa variation. S - Subject; LD - Latency Day; P Placental sample. 


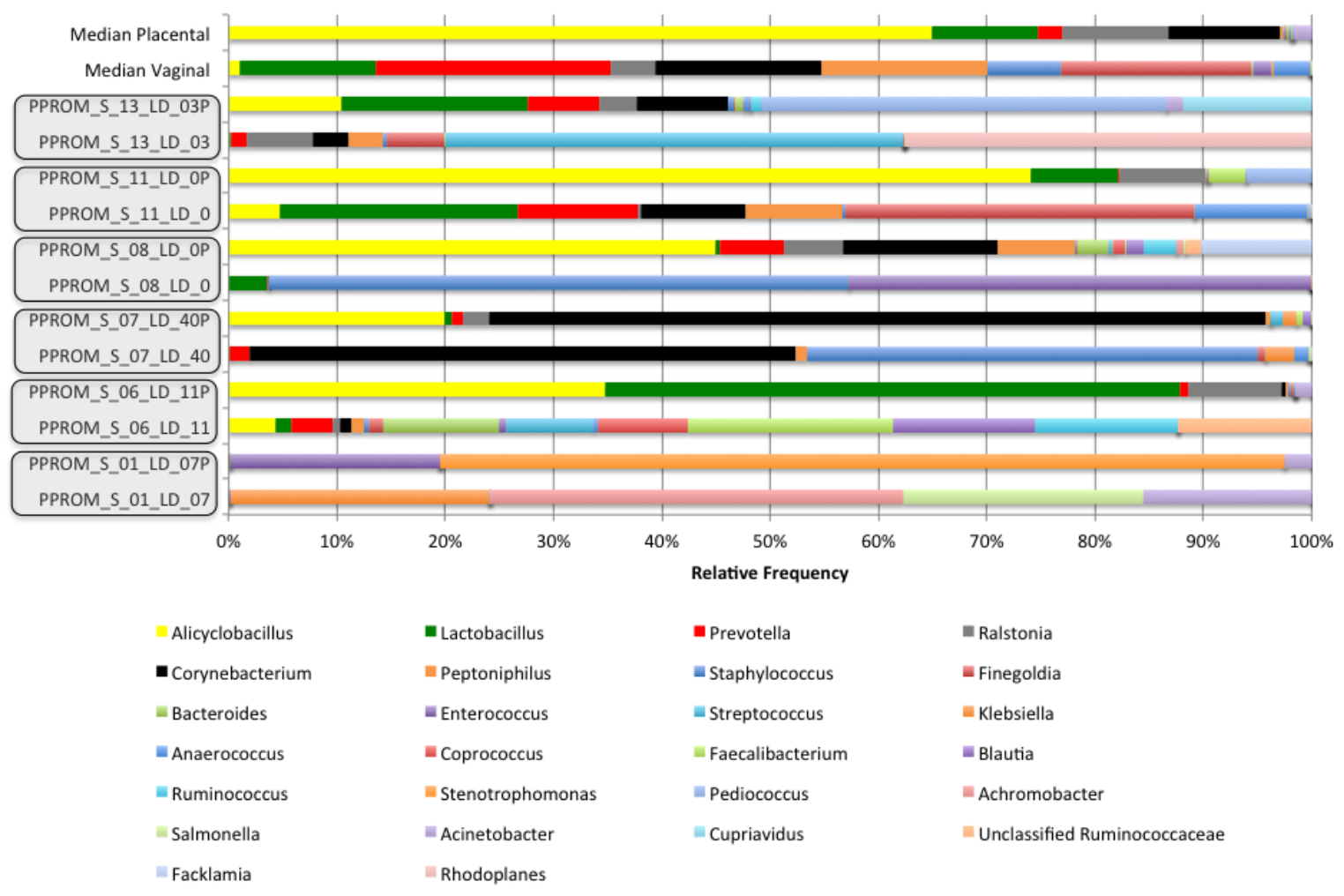

